\title{
Post Treatment Application of Jaungo after a Combined Therapy of Carbon Dioxide Laser and Trichloroacetic Acid in a Case of Vulvar Syringoma
}

\author{
En Hyung Kim ${ }^{1,2}$, Wonnam Kim ${ }^{3 *}$ \\ 1 Department of Dermatology, Cheil General Hospital and Women's Healthcare Center, Dankook University College of Medicine, \\ Cheonan, South Korea \\ ${ }^{2}$ Department of Dermatology, Bundang Jesaeng Hospital, Seongnam, South Korea \\ ${ }^{3}$ Division of Pharmacology, College of Korean Medicine, Semyung University, Jecheon, South Korea
}

\section{Key Words}

syringoma, jaungo, carbon dioxide laser

\begin{abstract}
Syringoma is a benign eccrine sweat gland tumor that predominantly appears in females during puberty with multiple smooth papules measuring 1-2 mm in diameter. Common locations are on lower eyelids and cheeks. Vulvar syringoma is quite a rare, occurring condition with only a few cases reported. Here, we are reporting a case of 31-year-old female with vulvar syringoma associated with pruritus. The lesion was treated with carbon dioxide (CO2) laser ablation and 50\% trichloroacetic acid (TCA) chemical peeling. Jaungo was used for wound care after laser abrasion. The combination treatment was effective for removing syringoma lesions. Post laser management with fusidic acid cream and jaungo cream was sufficient to prevent infection and promote wound healing.
\end{abstract}

\section{Introduction}

Syringoma is a tumor commonly seen in women often found as multiple, tiny, firm, skin- colored papules,

Received: May 21, 2019 Reviewed: Jul 15, 2019 Accepted: Sep 03, 2019

(c) This is an Open-Access article distributed under the terms of the Creative Common Attribution Non-Commercial License (http://creativecommons.org/licenses/by-nc/4.0/) which permits unrestricted noncommercial use, distribution, and reproduction in any medium, provided the original work is properly cited.

(1) This paper meets the requirements of KS X ISO 9706, ISO 9706-1994 and ANSI/NISO Z39.48-1992 (Permanence of Paper). originating from the eccrine sweat duct. They are usually located on the eyelid and the upper cheek [1]. However, syringomas involving the vulvar area has rarely been reported. It was first reported by Carneiro et al. in 1971 [2]. Because perspiration or increase in body heat tend to aggravate the pruritus associated with this entity, patients with vulvar syringomas usually first come to the clinic for evaluation of their symptoms rather than for cosmetic concerns $[3,4]$. Jaungo is a traditional topical medication, widely applied in Korea, Japan and China for various skin wounds and inflammatory skin diseases [5-7]. Jaungo mainly contains two herbs, the root of Lithospermum erythrorhizon Sieb. et Zucc. (Lithospermi Radix, LR) and the root of Angelica gigas Nakai (Umbelliferae) (Angelica Gigantis Radix, AR), and three carrier oils, sesame seed oil, bees wax, and swine oil [8]. Previous studies report the effects of jaungo on radiation dermatitis, atopic dermatitis, and wounded skin $[6,9,10]$. This report presents an unusual case of wide-spread syringomas on the vulva which was treated by carbon dioxide (CO2) laser ablation and trichloroacetic acid (TCA) chemical peeling. Also, jaungo was used for wound care after laser abrasion.

\section{Case Study}

A 31-year-old woman was presented to the outpatient department with a history of severe pruritus of 
the labia majora that persisted for several months. The lesions had appeared several months ago as "small bumps" and the number of lesions and size increased over the past few months. On physical examination, numerous yellow-brown pruritic papules on the vulva and the adjacent inner thighs were noted. Most of the papules were grouped together forming lichenified plaques or conglomerations (Fig. 1A). For symptom relief, she received oral antihistamines and topical corticoids for treatment, however the condition continued. Under the impression of prurigo, lichen planus, skin biopsy was performed. Histopathology revealed vulvar papules showing several tubular structures lined with rows of epithelial cells in fibrous stroma. In the papillary dermis, some dystrophic calcification or colloid material occurred in the lumina (Fig. 2). Based on clinical and histological characteristics the diagnosis for vulvar syringoma was confirmed. Although the patient wanted the lesions removed due to her symptoms, the lesions were located on her vulvar area and she was reluctant on having repeated sessions should the lesions recur. A $3 \times 3 \mathrm{~cm}$ sized lesion was tested first. The lesion was treated with a superpulse CO2 laser (SNJ1000 U Ultra laser, Korea). We used a focused, superpulsemode with power settings between $0.7 \sim 2$ watts. The spot size was $0.1 \mathrm{~mm}$ and pulse duration was 0.1 second. $50 \%$ TCA was applied to the laser irradiated site with wooden applicator stick, which was diluted with $3 \%$ boric acid after 2-3 minutes. After the procedure, fusidic acid an antibiotic ointment, was applied to the lesion. However, as the lesion was prone to irritations, such as friction, jaungo was also applied topically to speed up the healing process. Studies have shown that jaungo has wound healing and anti-inflammatory activities [5-7]. As there were no sign of infection or scarring, wider lesions were treated subsequently. Treatment for each side of the vulvar area was performed once at one week intervals. On follow-up after 8 weeks, the lesions were almost completely cleared with no subjective symptoms of pruritus. Only mild post inflammatory hyperpigmentation was noted (Fig. 1B).
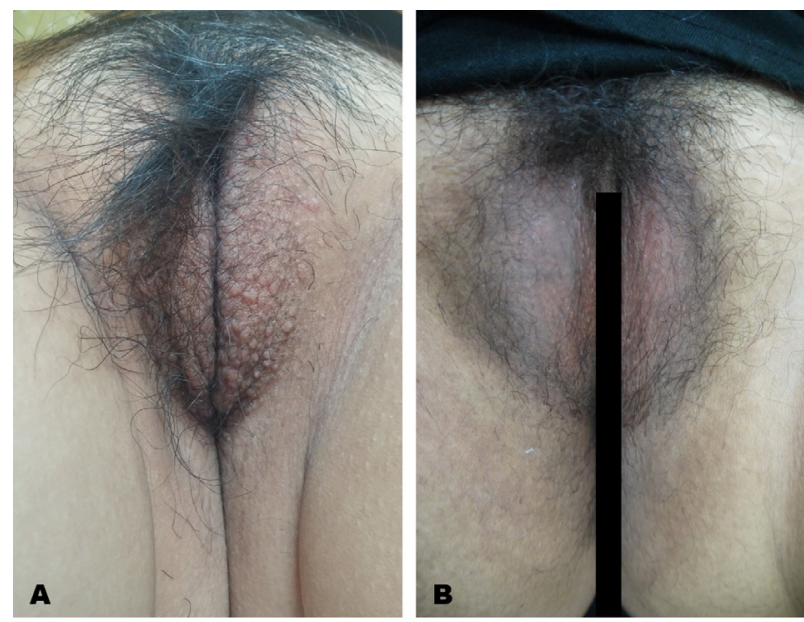

Figure 1 Clinical image before (A) and eight weeks after last treatment (B). A: Multiple yellow-brown-colored papules over labia majora, B: Good treatment response with few remaining papules and mild post inflammatory hyperpigmentation.

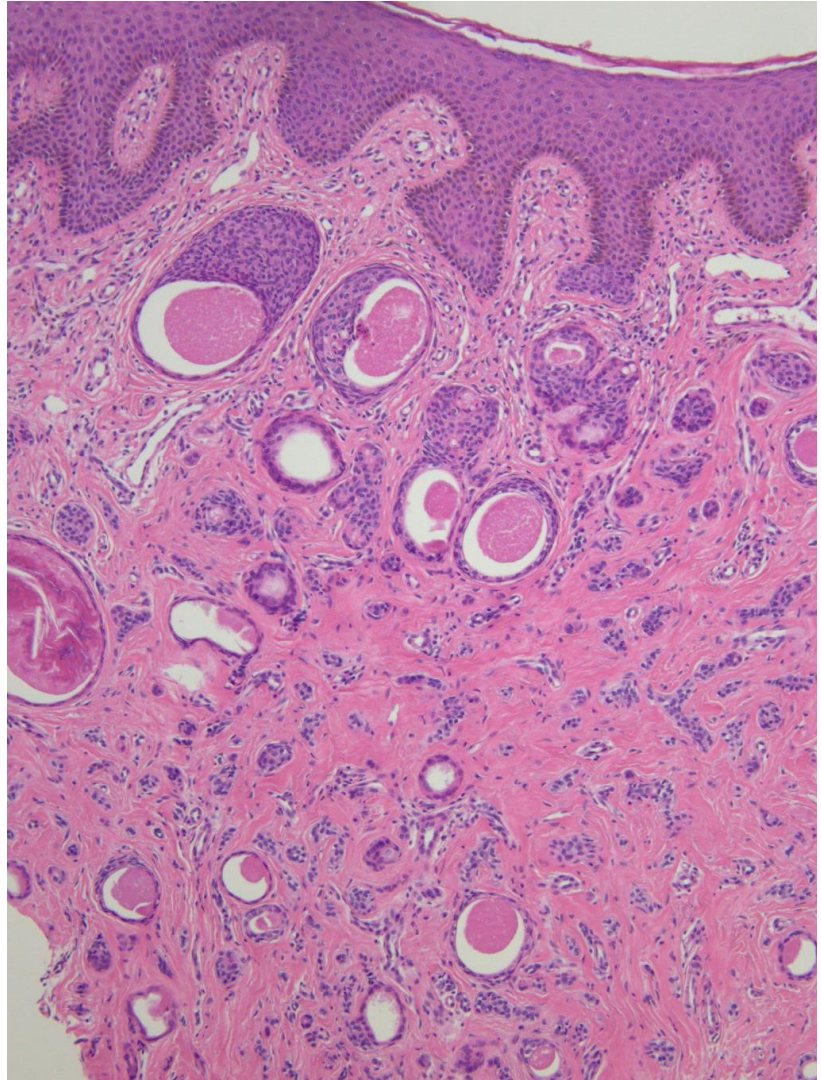

Figure 2 In the dermis, tubular structures lined with rows of epithelial cells contains some dystrophic calcification or colloid material in their lumina (Hematoxylin-eosin, x100).

\section{Discussion}

Syringomas do not usually present with accompanying symptoms, but sometimes pruritus can occur when the lesion involves the vulva area. During menstrual cycle, pregnancy, and the summer months, pruritic symptoms may likely be more severe [11]. The three clinical forms of vulvar syringomas presents as multiple flesh-colored or brownish papules with symmetrical involvement of the labia majora, cystic lesions or lichenoid plaques [11, 12]. The clinical differential diagnoses of note are steatocystoma multiplex, lichen planus, lichen simplex chronicus, condyloma, and epidermal cysts $[1,3,4,13]$.

As syringomas are benign tumors, treatment is usually not mandatory unless there are cosmetic concerns. Thus, the above mentioned patient was first treated with mild potent corticosteroid cream and oral antihistamines to relieve her pruritic symptoms however because of the remaining syringomas her symptoms frequently returned. On the other hand, treatment modalities for vulvar syringomas are not standardized and electro-desiccation, laser, cryotherapy resection, and excision are some of the methods that are performed with variable results [13-16]. One other option proven to be effective for the relief of pruritic symptoms and for safe and easy elimination of the lesions is carbon dioxide laser treatment $[11,15,16]$. Meanwhile, a study by Kang et al., have shown that applying 50\% TCA after CO2 laser irradiation effectively removed the 
deep-seated syringoma cells, and enhanced the cure rate [17]. Also, this combination method is known to be effective in reducing the bulk of the lesion [18]. Hence in order to remove the rather large lesions effectively with minimal side effects we used the above described combination method, which resulted in the clearing of the lesions.

In our patient's case, the lesions were located in the vulvar and inguinal areas which are prone to friction. Also, it was very difficult for the patient to apply covered dressings such as foam dressings or hydrocolloid dressings to protect and accelerate healing of the wound caused by CO2 laser and 50\% TCA. So for post-laser management, we applied fusidic acid ointment to prevent infection. And in order to expedite wound healing, we applied jaungo. In some incidences chemical peels reduce or remove skin appendages, which may impair epidermal regeneration causing delayed wound healing [19]. Delayed wound healing is known to cause hypertrophic scarring [20]. This may also be caused by prolonged inflammation induced by laser or chemical treatment leading to the activation of keratinocyte-secreted cytokines. These cytokines are involved in migration and deposition of fibroblasts in the matrix contributing to scar formation [21]. Park et al., reports that deoxyshikonin, an active component of LR, shows wound healing effects by promoting proliferation and migration in human keratinocytes through ERK and p38 MAPKs activation [22]. Moreover, studies have shown that jaungo has anti-inflammatory effects. Lu et al., demonstrated the anti-inflammatory action of shikonin, a substance isolated from LR [23]. Shikonin inhibits the release of TNF- $\alpha$, NF- $\chi$ B nuclear translocation, and proteasome activity in macrophage cells [23]. Also, Kim et al., showed that decursin, purified from AR, blunts NF- $\chi \mathrm{B}$ induced expression of inflammatory mediators in macrophages [24].

Although syringomas of the vulvar area are not an entity that is commonly diagnosed in clinical practice, this may be because lesions are sometimes asymptomatic and are located in private areas. However, these lesions can interfere with quality of life due to vulvar pruritus that is unresponsive to conventional treatments. In this case we showed that applying 50\% TCA after CO2 laser irradiation may be an effective method for removing troublesome vulvar syringomas. Also, jaungo was sufficient to promote wound healing on sensitive body areas where post-laser dressing was not possible.

\section{Conflicts of interest}

The authors declare no conflict of interest regarding the publication of this article.

\section{Patient consent}

Obtained.

\section{Funding/Support}

This paper was supported by the Semyung University Research Grant of 2017.

\section{References}

1. Dereli T, Turk BG, Kazandi AC. Syringomas of the vulva. Int J Gynaecol Obstet. 2007;99(1):65- 6.

2. Carneiro SJ, Gardner HL, Knox JM. Syringoma of the vulva. Arch Dermatol. 1971;103(5):494- 6.

3. Kopera D, Soyer HP, Cerroni L. Vulvar syringoma causing pruritus and carcinophobia: treatment by argon laser. J Cutan Laser Ther. 1999;1(3):181-3.

4. Gerdsen R, Wenzel J, Uerlich M, Bieber T, Petrow W. Periodic genital pruritus caused by syringoma of the vulva. Acta Obstet Gynecol Scand. 2002;81(4):369-70.

5. Hayashi M. Pharmacological studies on crude plant drugs, Shikon and Tooki (III) Effect of topical application of ether extracts and Shiunko on inflammatory reaction. Folia Pharmacologica Japonica. 1977;73(2):205-14.

6. Huang KF, Hsu YC, Lin CN, Tzeng JI, Chen YW, Wang JJ. Shiunko promotes epithelization of wounded skin. Am J Chin Med. 2004;32(3):389-96.

7. Lu PJ, Yang C, Lin CN, Li CF, Chu CC, Wang JJ, et al. Shiunko and acetylshikonin promote reepithelialization, angiogenesis, and granulation tissue formation in wounded skin. Am J Chin Med. 2008;36(1):115-23.

8. Yun Y, Ko Y, Ahn JH, Jang BH, Kim K, Ko SG, et al. Topical application of Jaungo in atopic dermatitis patients: study protocol for a randomized, controlled trial. Trials. 2017;18(1):176.

9. Higaki S, Kitagawa T, Morohashi M, Yamagishi T. Efficacy of Shiunko for the treatment of atopic dermatitis. J Int Med Res. 1999;27(3):143-7.

10. Kong M, Hwang DS, Lee JY, Yoon SW. The Efficacy and Safety of Jaungo, a Traditional Medicinal Ointment, in Preventing Radiation Dermatitis in Patients with Breast Cancer: A Prospective, Single-Blinded, Randomized Pilot Study. Evid Based Complement Alternat Med. 2016;2016:9481413.

11. Huang YH, Chuang YH, Kuo TT, Yang LC, Hong HS. Vulvar syringoma: a clinicopathologic and immunohistologic study of 18 patients and results of treatment. J Am Acad Dermatol. 2003;48(5):735-9.

12. Perez-Bustillo A, Ruiz-Gonzalez I, Delgado S, Alonso T, Ingelmo J. [Vulvar syringoma: a rare cause of vulvar pruritus]. Actas Dermosifiliogr. 2008;99(7):580-1.

13. Isaacson $\mathrm{D}$, Turner ML. Localized vulvar syringomas. J Am Acad Dermatol. 1979;1(4):352-6.

14. Karam P, Benedetto AV. Syringomas: new approach to an old technique. Int J Dermatol.1996;35(3):219-20.

15. Tay YK, Tham SN, Teo R. Localized vulvar syringomas--an unusual cause of pruritus vulvae. Dermatology. 1996;192(1):62-3.

16. Wang JI, Roenigk HH, Jr. Treatment of multiple facial syringomas with the carbon dioxide(CO2) laser. Dermatol Surg. 1999;25(2):136-9.

17. Kang WH, Kim NS, Kim YB, Shim WC. A new treatment for syringoma. Combination of carbon dioxide laser and trichloroacetic acid. Dermatol Surg. 1998;24(12):1370-4.

18. Frazier CC, Camacho AP, Cockerell CJ. The treatment of eruptive syringomas in an African American patient with a combination of trichloroacetic acid and CO2 laser destruction. Dermatol Surg. 2001;27(5):489-92.

19. McCollough EG, Langsdon PR, Seropian DM. Derma- 
brasion and Chemical Peel, A Guide for Facial Plastic Surgeons. Plast Reconstr Surg. 1990;86(1):155.

20. Okan G, Nouri K, Trent JS, Barbarulo AM, Rendon M. Delayed wound healing after laser resurfacing. Dermatol Surg. 2001;27(1):93-5.

21. McKay IA, Leigh IM. Epidermal cytokines and their roles in cutaneous wound healing. Br J Dermatol. 1991;124(6):513-8.

22. Park JY, Kwak JH, Kang KS, Jung EB, Lee DS, Lee S, et al. Wound healing effects of deoxyshikonin isolated from Jawoongo: In vitro and in vivo studies. J Ethnopharmacol. 2017;199:128-37.

23. Lu L, Qin A, Huang H, Zhou P, Zhang C, Liu N, et al. Shikonin extracted from medicinal Chinese herbs exerts anti-inflammatory effect via proteasome inhibition. Eur J Pharmacol. 2011;658(2-3):242-7.

24. Kim JH, Jeong JH, Jeon ST, Kim H, Ock J, Suk K, et al. Decursin inhibits induction of inflammatory mediators by blocking nuclear factor-kappaB activation in macrophages. Mol Pharmacol. 2006;69(6):1783-90. 